\title{
Fast Dump of the ATLAS Toroids
}

\author{
Alexey Dudarev, Gabriella Rolando, Giovanni Volpini, and Herman ten Kate
}

\begin{abstract}
The toroidal magnet system of the ATLAS Detector at CERN consists of a Barrel Toroid (BT) and two End Cap Toroids (ECT-A and ECT-C). Each toroid is built up from eight racetrack coils wound with an aluminum stabilized NbTi conductor and indirectly cooled by forced flow liquid helium. The three toroids operate in series at $20.5 \mathrm{kA}$ with a total stored energy of $1.5 \mathrm{GJ}$. In order to verify the reliability and effectiveness of the quench protection system, series of fast dump tests have been performed first of the single toroids and finally of the entire toroidal magnet system. In this paper a model to simulate the fast dump of the ATLAS toroids in single mode operation and in full system configuration is presented. The model is validated through comparison with measured data extracted from the ramp-and-quench runs. The calculated energy dissipation in the various coils is in very good agreement (within 1-2\%) with the enthalpy changes estimated from the temperature measurements of the different parts of the cold masses. The results confirm the safe operation of such a gigantic and complicated magnet system which is now ready for continuous running of the ATLAS Detector.
\end{abstract}

Index Terms-ATLAS, fast dump, superconducting magnet, toroid.

\section{INTRODUCTION}

$\mathbf{T}$ HE ATLAS Toroids feature three superconducting magnets connected in series to a common $20.5 \mathrm{kA}$ power supply (see Fig. 1). The design, fabrication and surface tests of the coils are already described in several papers [1]-[4]. The fast dump of the system is initiated by two simultaneous actions namely the opening of circuit breakers to disconnect the magnets from the power supply and the activation of quench heaters to fasten the normal zone propagation. Quench and fast discharge analysis for different operational scenarios have been performed during the $R \& D$ phase of the project [5].

Following the installation of the toroids in the ATLAS cavern, a new series of fast dump tests in standalone mode and in the complete configuration have been performed in order to verify the reliability of the protection scheme and study the coils response.

Table I summarizes the fast dump events (quenches and induced discharges) registered in the toroids since their installation in the ATLAS cavern. The good performance of the BT is opposed to the ECTs ones where a number of training quenches has taken place. Training quenches are observed in the End Cap

Manuscript received October 19, 2009. First published March 25, 2010; current version published May 28, 2010. This work was supported by the ATLAS Collaboration, presently about 2200 scientific authors from 164 institutes in 35 countries and supported in part by CERN.

A. Dudarev and H. ten Kate are with the European Center for Nuclear Research-CERN, 1211 Geneva CH, Switzerland (e-mail: alexey.dudarev@cern. ch).

G. Rolando is with University of Torino, Torino 10121, Italy.

G. Volpini is with LASA Laboratory, INFN, Milano 20090, Italy.

Color versions of one or more of the figures in this paper are available online at http://ieeexplore.ieee.org.

Digital Object Identifier 10.1109/TASC.2010.2042695

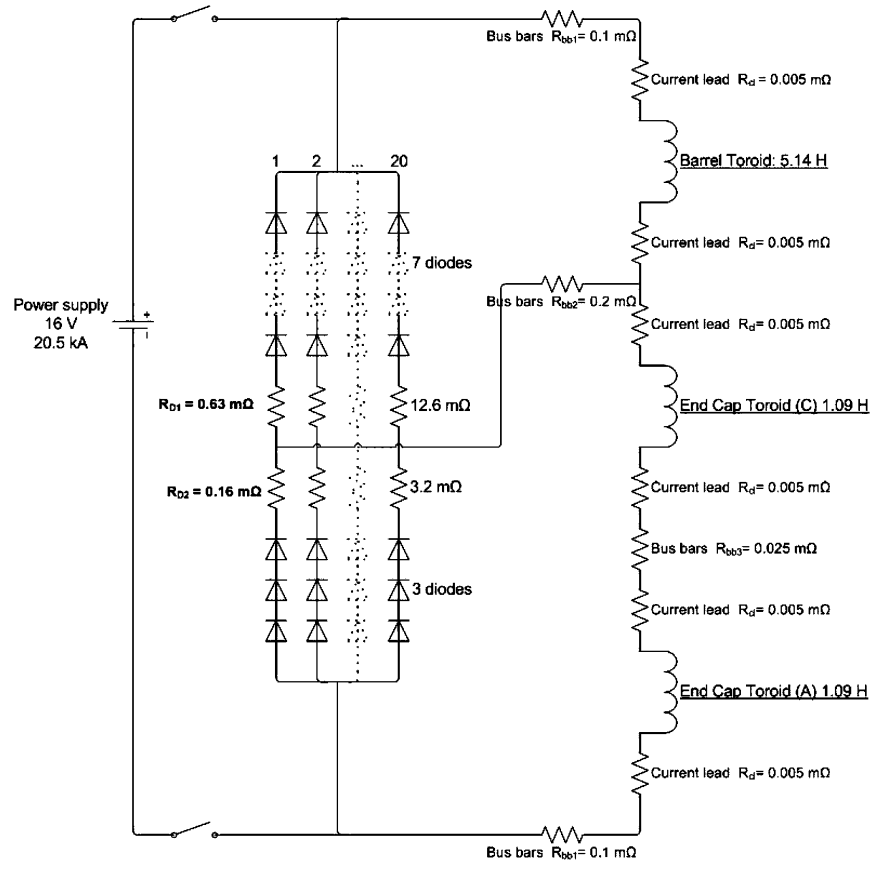

Fig. 1. Electrical circuit of the ATLAS toroidal magnet system.

TABLE I

ATLAS TOROIDS FAST DUMP SUMMARY

\begin{tabular}{cccc}
\hline \hline Configuration & $\begin{array}{c}\text { N. of Fast } \\
\text { Dumps }\end{array}$ & $\begin{array}{c}\text { Training } \\
\text { Quenches }\end{array}$ & $\begin{array}{c}\text { Induced } \\
\text { Fast Dump }\end{array}$ \\
\hline BT & 7 & 0 & 7 \\
ECT-A & 8 & 4 & 4 \\
ECT-C & 6 & 3 & 3 \\
BT + ECTs & 6 & 3 & 3 \\
\hline \hline
\end{tabular}

Toroids even after having reached the nominal current. No differences in the voltage and current evolution during a fast discharge are noticed between the operation of the magnets in the stand alone and complete configurations.

In this paper the electrical and thermal behavior of the magnets during a fast discharge is simulated and the magnetic field energy final distribution calculated for the different tested configurations.

\section{FAST Dump of the ATLAS Toroids IN Single Mode}

\section{A. Electrical Model}

During the operation of each toroid in single mode the other two magnets and their dump units are by-passed through cables. The magnetic coupling of the coils to their casings, thermal shields and vacuum vessels is ignored as the energy dissipated as eddy currents in the above elements during a fast discharge is verified to be a negligible fraction of the initial one (lower than $1 \%$ per coil). To further simplify the model the voltage drop across each diode of the dump unit is assumed constant and 
TABLE II

TOROIDS MAIN ElECTRICAL PARAMETERS

\begin{tabular}{|c|c|c|c|}
\hline Magnet & Name & Value & Units \\
\hline \multirow[t]{3}{*}{ BT } & $\mathrm{L}_{\mathrm{BT}}$ & 5.14 & $\mathrm{H}$ \\
\hline & $\mathrm{R}_{\mathrm{BT}}$ & 0.97 & $\mathrm{~m} \Omega$ \\
\hline & $\mathrm{V}_{\mathrm{D} \_\mathrm{BT}}$ & 4.9 & V \\
\hline \multirow[t]{3}{*}{ ECT } & $\mathrm{L}_{\mathrm{ECT}}$ & 1.09 & $\mathrm{H}$ \\
\hline & $\mathrm{R}_{\mathrm{ECT}}$ & 0.37 & $\mathrm{~m} \Omega$ \\
\hline & $V_{\text {D_ECT }}$ & 2.1 & $\mathrm{~V}$ \\
\hline
\end{tabular}

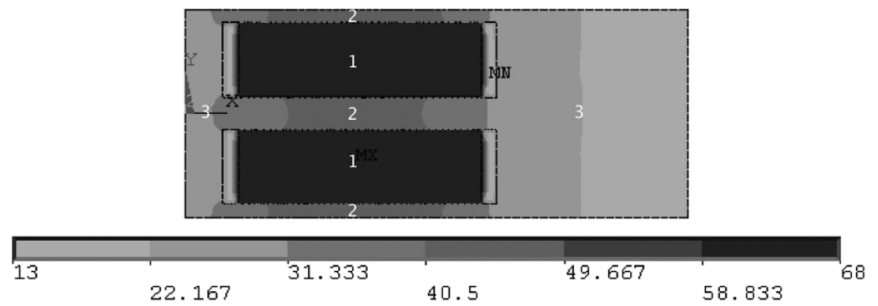

Fig. 2. Temperature profile (in Kelvin) in a BT casing cross section after $120 \mathrm{~s}$ from the beginning of a fast dump from $20.5 \mathrm{kA}$.

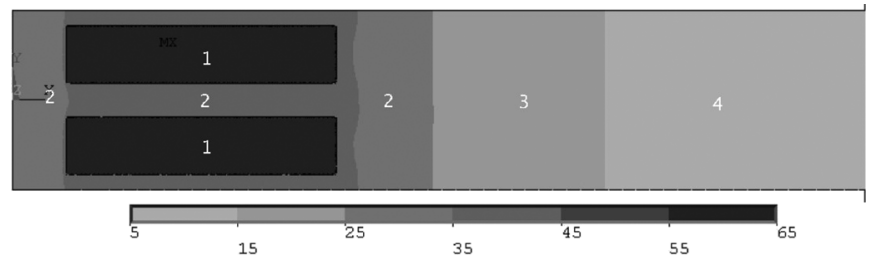

Fig. 3. Temperature profile (in Kelvin) in an ECT casing cross section after $60 \mathrm{~s}$ from the beginning of a fast dump from $20.5 \mathrm{kA}$.

equal to $0.7 \mathrm{~V}$. With this assumption the equation governing the current decay results to be

$$
L_{i} \cdot \frac{d I}{d t}+I \cdot\left(R_{i}+R_{\text {coils }}\right)+V_{D \_i}=0
$$

with $\mathrm{L}$ self inductance of the magnet, $\mathrm{R}$ equivalent resistance of the cables and the dump unit resistors and $V_{D}$ voltage drop across the diodes of the dump unit (see Table II).

\section{B. Thermal Model}

Besides the Joule effect, a second thermal process taking place inside the coils during the fast dump is the diffusion to the casing of the heat generated inside the conductor. Given the casing dimensions and the $\mathrm{Al}$ alloy heat diffusivity, the resulting temperature increase is not uniform and a thermal gradient sets up inside the casing. The heat dissipation and diffusion processes have been analysed through a FEA simulation (see Fig. 2) on the base of which the coil cross section has been divided in three regions having different thermal evolution during the discharge.

The formation of the thermal gradient is more relevant for the ECTs than for the BT due to the presence of a central Al alloy plate that totally occupies the center of the coils. As a consequence of the increased width of the casing the coil cross section needs to be subdivided in a higher number of regions (see Fig. 3).

\section{Conductor Resistivity \& Initial Conditions}

Since $\mathrm{Al}$ resistivity is much lower than $\mathrm{NbTi}$ once in the normal state, as soon as the windings get a resistance current dif-

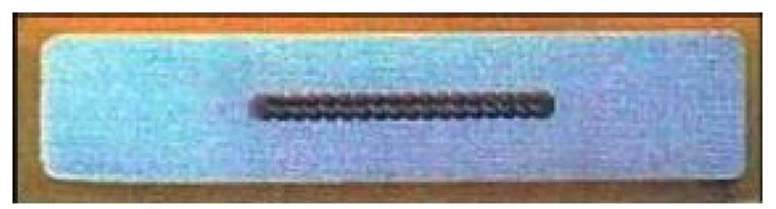

Fig. 4. ATLAS barrel toroid conductor. A $32 \mathrm{Cu} / \mathrm{NbTi}$ strands Rutherford cable is surrounded by high purity aluminum stabilizer.

TABLE III

MAGNETIC ENERGY DENSITY

\begin{tabular}{ccccc}
\hline \hline Magnet & Material & $\begin{array}{c}\text { Cross section } \\
\left(\mathbf{m ~ m}^{\mathbf{2}}\right)\end{array}$ & $\mathbf{I}(\mathbf{k A})$ & $\begin{array}{c}\mathbf{E} / \mathbf{I} \\
(\mathbf{k J} / \mathbf{m})\end{array}$ \\
\hline BT & Sc Cable & 18.5 & 20.5 & 7.10 \\
& Al & 632 & 20.5 & 6.95 \\
\multirow{2}{*}{ ECT } & Sc Cable & 18.5 & 20.5 & 6.85 \\
& Al & 440 & 20.5 & 6.75 \\
\hline \hline
\end{tabular}

TABLE IV

MAGNETIC ENERGY RELEASE IN THE CONDUCTOR

\begin{tabular}{ccccc}
\hline \hline Magnet & $\begin{array}{c}\mathbf{\Delta E} / \mathbf{l} \\
(\mathbf{k J} / \mathbf{m})\end{array}$ & $\begin{array}{c}\text { Cable length } \\
(\mathbf{k m})\end{array}$ & $\begin{array}{c}\text { Released } \\
\text { energy (MJ) }\end{array}$ & $\begin{array}{c}\text { Cable } \\
\text { temperature } \\
(\mathbf{K})\end{array}$ \\
\hline BT & 0.2 & 59 & 10 & 21 \\
ECT & $\mathrm{NbTi}$ & 12.8 & 1.3 & 19 \\
\hline \hline
\end{tabular}

fuses from the cable to the surrounding stabilizer (see Fig. 4). Apart from temperature, the main parameters affecting the $\mathrm{Al}$ resistivity are the RRR and the magnetic field. For the BT the average magnetic field has been calculated with a software specifically developed for the reconstruction of the magnetic field distribution in the ATLAS detector [6] and is found to be $1.8 \mathrm{~T}$; while for the ECT conductor a FEA analysis has been performed which gives a result of $2 \mathrm{~T}$.

The current diffusion in super-stabilized conductors as the ATLAS toroids cable is already described in several papers [7], [8]. In the present work the attention has been focused on the energy released to the conductor due to the changing field distribution produced by the current diffusion. Assuming the entire current flow in the superconductor in the beginning and in the $\mathrm{Al}$ stabilizer at the end of the diffusion process, the magnetic energy density per meter length of conductor is calculated through a FEA analysis (see Table III).

Knowing the conductor length, the energy release is obtained from the difference of the energy densities in the initial and final configurations (see Table IV). Through the conductor enthalpy this energy is verified to produce a significant increase of the temperature of the cable above the NbTi critical temperature, with the result that the coils can be considered completely resistive from the beginning while the propagation of the normal zone can be neglected.

\section{Barrel Toroid Results}

The simulation of the fast dump is performed through a numerical method implemented in Mathematica evaluating the resistance of the coils, the current, the Joule dissipation in the coils, the heat transfer to the casing and the temperature increase of the system. The electrical results of the simulation are shown in Fig. 5.

The most critical part can be recognized in the beginning of the discharge as the model does not consider the normal zone 


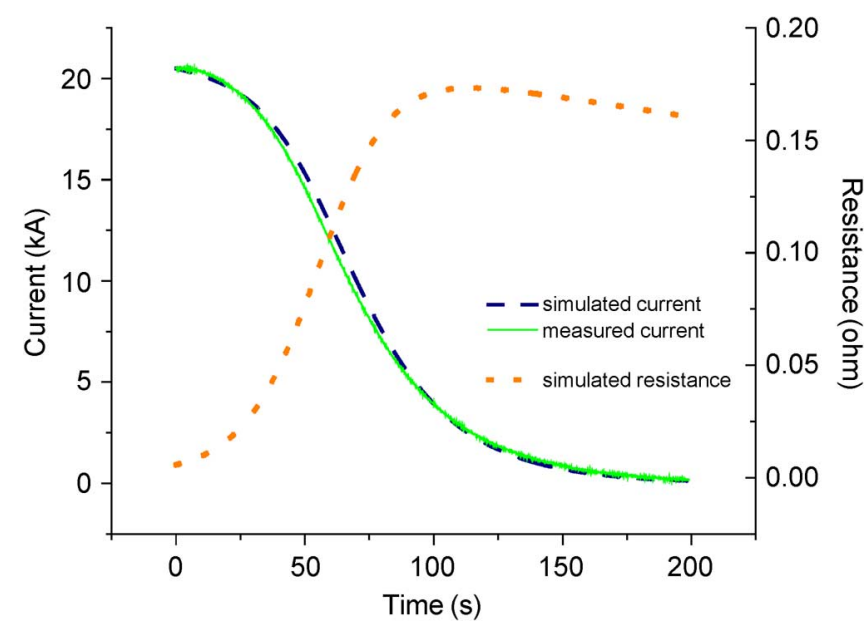

Fig. 5. BT current and resistance evolution during a fast dump is stand alone mode from $20.5 \mathrm{kA}$.

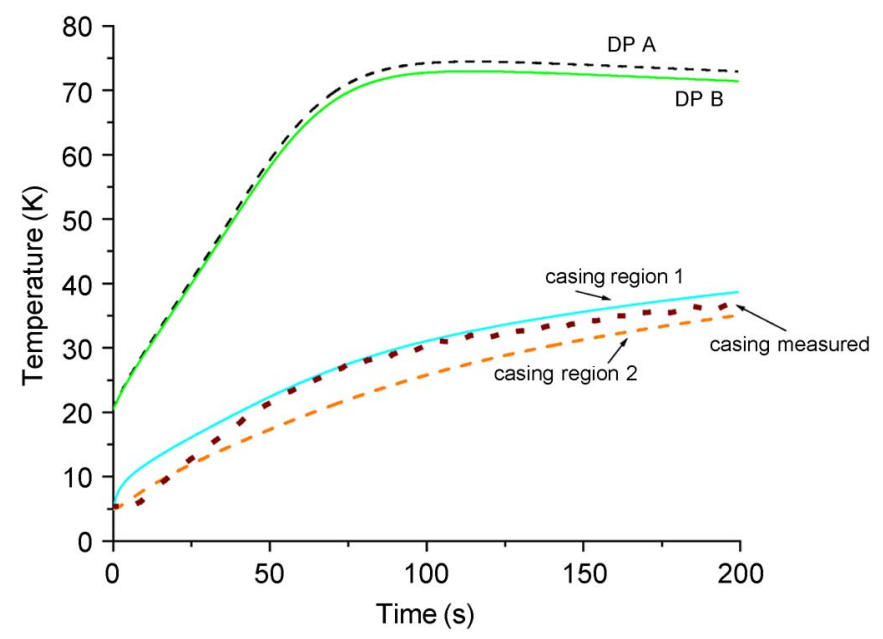

Fig. 6. Simulated (dashed line) and measured temperature evolution for the DPs (double pancakes) and casing from the beginning of the dump.

propagation. Moreover the initial phase is the most affected by the accuracy of the magnetic field value. In spite of the simplifications assumed, the model proves to be able to describe the real current behavior with an adequate level of accuracy. The maximum value reached by the BT resistance is lower than $200 \mathrm{~m} \Omega$ which ensures a safe discharge of the magnet.

An example of the temperature evolution of the coils and the casing can be seen in Fig. 6. The simulated maximum temperature of the BT double pancakes in the case of a $20.5 \mathrm{kA}$ fast dump induced by the simultaneous activation of all the heaters is lower than $80 \mathrm{~K}$ which ensures the absence of risks for the cable integrity. When the simulated temperatures are compared to the measured ones at the end of the dump and to the temperature of the windings estimated on the base of the energy dissipation, the average difference is found to be $1 \%$ corresponding to an accuracy of $\sim 0.7 \mathrm{~K}$, which is equal to the accuracy of the employed Pt1000 thermometers in the considered temperature range.

\section{E. BT Energy Distribution}

Since the BT operating pressure is $\sim 10^{-6} \mathrm{~Pa}$, the heat transfer from the casing to the surrounding environment can be considered equal to zero and the energy dissipated in the
TABLE V

BT ENERGY DISTRIBUTION AT FAST DUMP

\begin{tabular}{ccc}
\hline \hline Element & $\begin{array}{c}\text { Dissipated energy } \\
\text { (MJ) }\end{array}$ & $\begin{array}{c}\text { \% } \\
\text { Initial energy }\end{array}$ \\
\hline Al stabilizer & 299 & 28 \\
Cu/NbTi & 54 & 5 \\
Insulation & 160 & 15 \\
Casing & 518 & 48 \\
Dump unit & 33 & 3 \\
\hline \hline
\end{tabular}

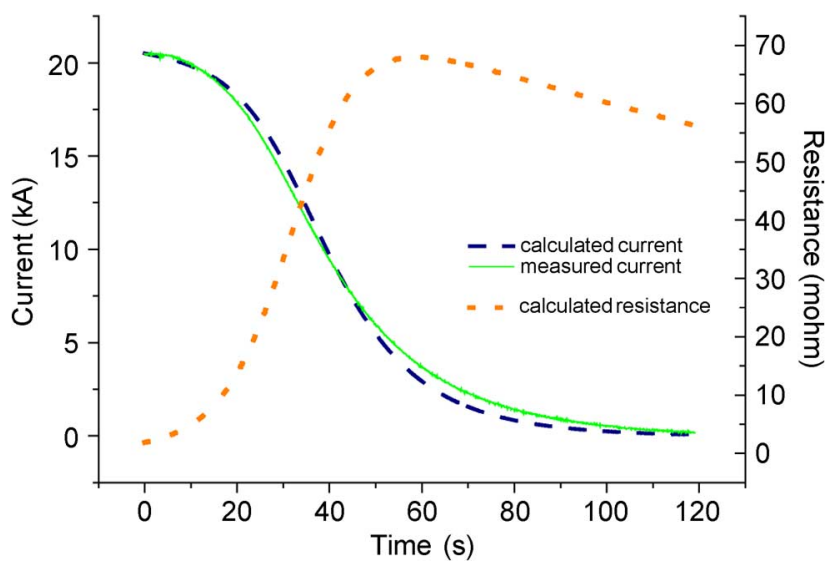

Fig. 7. ECT-A current and resistance evolution during a fast dump in standalone mode from $20.5 \mathrm{kA}$.

conductor remains stored inside the coils at the end of the dump being shared among the constituent materials. In total $96 \%$ of the initial energy of the magnetic field is stored in the coils at the end of the discharge, another $3 \%$ being dissipated in the dump unit (Table V).

The remaining missing energy is explained considering the employed temperatures are lowered by the coolant flow which is re-started before the coils have reached thermal equilibrium; moreover the contribution of the ribs in the coil casing has not been included in the calculation. The present result proves the fast dump to be an internal discharge mode and that the BT coils can be considered as adiabatic systems.

\section{F. End Cap Toroid-A Results}

Fig. 7 shows the result of the electric simulation. In spite of neglecting the normal zone propagation, the simulated curve matches quite accurately the real current evolution until $50 \mathrm{~s}$ from the start. The fastest decay rate in the last part of the dump is likely to be due to the influence of support structures (keystone boxes) against the magnetic field forces placed between the coils. Since the keystone boxes are in thermal contact with the coils a heat transfer exists which lowering the winding temperatures produces a slower current decay than in an adiabatic system, as assumed by the model.

The maximum temperature reached by the coils in the case of a $20.5 \mathrm{kA}$ fast dump initiated by the simultaneous activation of all the quench heaters is slightly higher than for the BT value being lower than $82 \mathrm{~K}$. The difference between the simulated temperatures and the real measurements also results to be higher $(\sim 3.5 \%)$ because of the heat transfer proceeding from the coils to the keystone boxes. The final measured temperatures are also lowered by the helium flow re-start before the achievement of thermal equilibrium. 
TABLE VI

ECT-A ENERGy DisTRIBUTION AT FAST DUMP

\begin{tabular}{ccc}
\hline \hline Element & $\begin{array}{c}\text { Dissipated energy } \\
\text { (MJ) }\end{array}$ & $\begin{array}{c}\% \\
\text { Initial energy }\end{array}$ \\
\hline Al stabilizer & 24 & 11 \\
Cu/NbTi & 7 & 3 \\
Insulation & 7 & 3 \\
Casing & 147 & 64 \\
Keystone boxes & 26 & 11 \\
Dump unit & 9 & 4 \\
\hline \hline
\end{tabular}

\section{G. End Cap Toroid-A Energy Distribution}

The main storing element is the coil casing with almost $2 / 3$ of the initial energy. Another important contribution is the keystone boxes, which confirms the ECT coils cannot be considered adiabatic, but the support structures have a relevant role in the energy balance of the magnet. The missing energy is $4 \%$ and is likely to be caused by the usage of temperatures lowered by the re-start of the coolant circulation (Table VI).

\section{FAST DUMP OF THE COMPLETE ATLAS TOROID SySTEM}

Finally the models developed for the single magnets have been unified to simulate the fast dump of the entire toroid system in the operational configuration. The complete electrical circuit is shown in Fig. 1.

No relevant differences in the electric results of the simulation can be observed between the single mode and complete configuration operations.

The average difference between simulated and measured BT temperatures at thermal equilibrium doubles in the complete toroid system. The result is due to an average increase of the measured values of $1 \mathrm{~K}$ which cannot be explained on the base of the coil energy dissipation as this is observed not to change between the two configurations. The behavior results more difficult to explain as, on the other side, temperatures measured after $200 \mathrm{~s}$ from the beginning of the dump in the complete configuration are found to be more than $2 \mathrm{~K}$ lower than in a BT single mode discharge. In the actual state the observations are attributed to internal energy transfer phenomena between the toroids, idea also supported by the increased energy dissipation registered in the ECT-A coils in the complete configuration (average increment $\sim 3 \mathrm{MJ}$ ).

At the end of $20.5 \mathrm{kA}$ fast dump more than half of the energy is stored in the casings which are the most massive elements. Important contributions also come from the $\mathrm{BT} \mathrm{Al}$ stabilizer and insulation, while all the other parts contribute for less than $2 \%$ each. Although having a reduced importance on the total system energy balance the keystone boxes are observed to store a not negligible fraction of energy. The missing energy is mainly due
TABLE VII

ATLAS TOROID ENERGY DISSIPATION

\begin{tabular}{cccc}
\hline \hline \multicolumn{2}{c}{ Element } & $\begin{array}{c}\text { Dissipated energy } \\
\text { (MJ) }\end{array}$ & $\begin{array}{c}\text { \% } \\
\text { Initial energy }\end{array}$ \\
\hline BT & Al stabilizer & 323 & 20 \\
& Cu/NbTi & 58 & 4 \\
& Insulation & 168 & 11 \\
\multirow{6}{*}{ ECTs } & Casing & 560 & 35 \\
& Al stabilizer & 46 & 3 \\
& Cu/NbTi & 13 & 0.8 \\
& Insulation & 13 & 0.8 \\
& Casing & 279 & 18 \\
Dump unit & Key. boxes & 60 & 4 \\
\hline \hline
\end{tabular}

to the error on the final temperatures which are lowered by the helium flow re-start (Table VII).

\section{CONCLUSION}

The fast dump of the three ATLAS toroids in single mode and in the complete configuration has been simulated. The models implemented have been proven to be able to describe the electric and thermal behavior of the system with a good level of accuracy in spite of the simplifications assumed. Through the calculation of the energy dissipation in the coils the fast dump is verified to be an internal discharge mode. Finally the energy distribution at the end of the discharge shows the BT coils to be an adiabatic system, while the energy balance of the ECTs is strongly influenced by the presence of keystone boxes.

\section{REFERENCES}

[1] A. Dudarev, J. J. Rabbers, C. Berriaud, S. Junker, R. Pengo, P. Vedrine, H. J. J. ten Kate, E. Adli, M. Arnaud, F. Broggi, and G. Volpini, "On surface test of the ATLAS barrel toroid coils: Overview," IEEE Trans. Applied Superconductivity, vol. 16, no. 2, pp. 557-560, Jun. 2006.

[2] A. Dael et al., "Construction of the ATLAS B0 model coil," IEEE Trans. Applied Superconductivity, vol. 11, no. 1, pp. 1597-1600, 2001.

[3] H. H. J. ten Kate, "ATLAS superconducting toroids and solenoid," IEEE Trans. Applied Superconductivity, vol. 15, no. 2, pp. 1267-1270, 2005.

[4] D. E. Baynham, F. S. Carr, E. Holtom, J. Buskop, A. Dudarev, G. Vandoni, R. Ruber, A. Foussat, M. Losasso, P. Benoit, R. Pengo, L. Stewart, A. Olyunin, V. Stepanov, N. Kopeykin, I. Shugaev, M. Arnaud, and H. H. J. ten Kate, "ATLAS end cap toroid final integration, test and installation," IEEE Trans. Applied Superconductivity, vol. 18, pp. 391-394, 2008 .

[5] ATLAS Barrel Toroid Technical Design Report ATLAS TDR 7, 1997, CERN/LHCC/97-19.

[6] L. Chevalier, Magnetic Field Reconstruction May 2005 [Online]. Available: https://twiki.cern.ch/twiki/bin/view/Atlas/MagneticFieldReconstruction

[7] A. Gavrilin and Y. M. Eyssa, "Modeling of electromagnetic and thermal diffusion in a large aluminium stabilized superconductor under quench," IEEE Trans. Applied Superconductivity, vol. 2, no. 1, pp. 2559-2602, Mar. 2001.

[8] A. Gavrilin, A. Dudarev, and H. H. J. ten Kate, "Quench modeling of the ATLAS superconducting toroids," IEEE Trans. Applied Superconductivity, vol. 2, no. 1, pp. 557-560, Mar. 2001. 